
\title{
25 Research Soure \\ Error analysis of a robot positioning system based on biplanar navigation technology
}

\section{Hailong Du}

Department of Orthopaedics, Chinese PLA General Hospital

\section{Ning Hu}

Department of Mechanical, Aerospace and Biomedical Engineering, University of Tennesee

\section{Guoxin Yu}

Robits Institute, Beihang University

Hongsheng Liu

Robotics Institute, Beihang University

Shuwei Zhang

Chinese PLA General Hospital

\section{Gongzi Zhang}

Department of Rehabilitation and Physiotherary, Chinese PLA General Hospital

\section{Jian Zhang}

Robotics Institute, Beihang University

\section{Jindong Tan}

Department of Mechanical, Aerospace and Biomedical Engineering, University of Tennessee

\section{Peifu Tang}

Department of Orthopardics, Chinese PLA General Hospital

\section{Lihai Zhang ( $\nabla$ zhanglihai74@qq.com )}

Department of Orthopedics, Chinese PLA General Hospital https://orcid.org/0000-0003-3172-6309

\section{Research article}

Keywords: Image intensifier, Biplanar navigation, Conical distortion, Respiratory oscillation, Image drift

Posted Date: February 20th, 2020

DOI: https://doi.org/10.21203/rs.2.24100/v1

License: (9) This work is licensed under a Creative Commons Attribution 4.0 International License. Read Full License 


\section{Abstract}

Background: Some problems have been found in the clinical application of biplanar navigation technology. In particular, conical distortions caused by the X-ray image intensifier and deleterious effects on the navigation image caused by respiratory motion are occasionally ignored by surgeons. This paper discusses possible errors in the practical application of biplanar navigation technology and performs an analysis of the experimental correction for the conical distortion caused by X-rays.

Methods: Five patients with unstable fractures of the pelvic posterior ring were included in this study. In four of these cases, the patient received a sacroiliac screw placement using biplanar navigation technology, while one received a respiratory motion test. The standard pelvic inlet and outlet positions were used for positioning precision verification. Using the images and manual tests, the point positioning error was measured and analyzed, and the image distortion was corrected by means of a geometric transformation mapping. A circle was fitted to the corrected lines, and the radius of this circle was used to evaluate the correction.

Results: The preoperative planning agreed with the sacroiliac screw placement performed using biplanar navigation technology (representing precise positioning) in only one of the four cases. The other three cases presented deviations. Because of the possibility to cut out the nailing channel, the positioning operation was converted to a manual method. The breathing motion test showed that the pelvis can vibrate back and forth with breathing movement, especially the sacral promontory, with a maximum amplitude above $5 \mathrm{~mm}$. The positioning error of the points in space with the biplanar technology was within $2 \mathrm{~mm}$ (entrance point, $1.60 \pm 0.28 \mathrm{~mm}$; exit point, $1.28 \pm 0.43 \mathrm{~mm}$ ). The corrected image curvature was significantly reduced, and the error can be greatly reduced.

Conclusions: Conical distortion error and respiratory motion can affect the accuracy of biplanar navigation systems.

\section{Background}

With the development of computer technology, a large number of computer-aided surgery (CAS) techniques have emerged in the medical field, with these techniques being mainly concerned with the functions of positioning, preoperative planning, and intraoperative auxiliary manipulation[1]. In recent years, CAS technology has shown some outstanding characteristics, and its technical advantages have become increasingly apparent. However, computer technology emanates from the engineering field and may suffer from many limitations when applied to the clinical field.

In the present medical field, CAS technology can be largely divided into soft tissue manipulation robots and hard tissue navigation robots. Soft tissue robots act as extensions of the doctors' hands and eyes, mainly using endoscopic technology. They use optical and mechanical technology to perform surgery using minimally invasive access, and are used in thoracic surgery, general surgery, urology, gynecology, and laryngology, in combination with laparoscopy, thoracoscopy, arthroscopy and hysteroscopy (such as 
the DaVinci and Zeus surgical robot)[2-7]. The technology in these surgical robots is relatively mature, and they have been available on the market for many years. Hard-tissue robots make use of the high contrast of certain body tissues on radiological imaging, such as that of bone on X-ray-based imaging, to conduct image processing and reconstruction, and include biplanar navigation robots and spine and joint surgery robots [8-10].

For minimally invasive operations in orthopedics, omni-directional and multi-angle perspectives are needed to determine the nailing channel and to avoid the iatrogenic injury of important soft tissue such as the spinal cord and the neurovasculature in front of the pelvic posterior ring [10, 11]. In such minimally invasive procedures, the patient and surgeon may be exposed to a relatively large radiation dose.

CAS technology can digitally reconstruct the bone structure through image processing technology, allowing the important soft tissues to be protected by using the navigation technology to determine the nailing channel both preoperatively and intraoperatively. In the practical application of CAS technology, small errors may come from many sources. Sometimes the accumulated error reaches the level of image drift, namely errors between the virtual position and posture and the actual ones in the skeleton, and it may be difficult to attract the attention of the operator to such problems. Once image drift occurs, the system should be reregistered, and the whole procedure should be recommenced from the beginning, which can greatly prolong the operation time [10]. If the image drift is ignored, there is a great possibility of serious iatrogenic damage to the patient.

In clinical work, a biplanar positioning robot system is frequently used to locate the sacroiliac nailing channel. However, the operative procedure is subject to several problems. First, when the positioning images are acquired by the biplanar robot, there are typically differences due to individual differences, projection angle, and the subjective intention of the operators. Second, the X-rays emitted by the X-ray tube are not parallel, but are in a conical configuration. The dispersion extent of the X-rays also differs according to the different projection planes, and the images of complex structures can be distorted [1214]. Finally, as abdominal pressure fluctuates in association with respiratory movement, the spatial posture of the pelvis can show a slight anteversion at the end of inhalation compared with the end of exhalation, and this posture can oscillate at any time during the operation process.

In this paper, we analyzed the sources of error in biplanar navigation technology through the establishment of a model, to help clinical workers to further understand the technology and its application to clinical work.

\section{Materials And Methods}

1. Clinical research

\subsection{Patient group}


Five patients were enrolled in this study, all of whom suffered from a high-energy injury. Four cases underwent sacroiliac fixation, including B1-type (tile classification) sacroiliac joint separation, with three cases being on the right side and one on the left side. The other patient was evaluated for respiratory oscillation.

\subsection{Data Collection}

For the patients undergoing sacroiliac fixation, the biplanar navigation system was used for sacroiliac fixation, with standard procedures for positioning, filming, registration, planning, and fixation placement. Standard pelvic inlet and outlet films were used for positioning (as shown in Fig. 1-4). During the positioning procedure using the biplanar navigation system, the inlet and outlet films are intermittently viewed and compared with those of the preoperative planning images. If there is no deviation, the fixation procedure is continued. If there is deviation, the incidence of the iatrogenic injury is evaluated according to the operator's experience. If there is unlikely to be any serious consequence and the positioning is acceptable, the fixation procedure is continued. However, if an iatrogenic injury may occur, the fixation procedure is transferred to a manual method.

In the evaluation of respiratory oscillation, the patient was maintained under general anesthesia and the endotracheal intubation, and image intensifier were kept still. Five consecutive fluoroscopies were taken at the inlet and outlet position at intervals of 2-3 s. Variations in the positions of characteristic anatomical structures (such as the sacrum front, sacrum promontory, and the great notch of ischia) were observed.

\section{The Error Verification Experiment}

By means of the biplanar navigation, the spatial positioning errors of the tips of Kirschner wires were measured. Two Kirschner wires were fixed on the bottom plate of the navigation system, and the actual coordinates of the tips were measured with a height ruler and Vernier caliper, and were considered as the true values. The inlet and outlet films were then acquired, and the Kirschner wire tips were taken as the targets for the positioning calculation. Using three-dimensional measurement technology based on the parallax method, the calculated values of their coordinates were obtained. The difference between the true values and the calculated values was then calculated as the positioning error of the system.

\section{The Error Calibration Experiment}

A rectangular block of $50 \times 80 \mathrm{~mm}$ was manufactured from Polyvinyl chloride (PVC), as shown in Fig. 5 . Three Kirschner wires of a length of $100 \mathrm{~mm}$ and a diameter of $1.5 \mathrm{~mm}$ were embedded into the block at spacings of $30 \mathrm{~mm}$. A 50-mm length of the wires was embedded into the block from its long edge. Whilst assuring that the component was horizontal to the receiving end of the image intensifier, five perspectives 
were acquired at distances of 150, 200, 250,300, and $350 \mathrm{~mm}$ from the receiving end. Nine pixels from A-I were selected on the axis of the Kirschner wires before and after image correction, with the pixels $B, E$, and $\mathrm{H}$ being at the other long edge of the block and also the centers of $A C, D F$, and $\mathrm{Gl}$, as shown in Fig. 5C. The actual scale of the image was calculated from line $E F$, and the lengths of $A D, D G, B E, E H, C F$, and $F I$ were then calculated. The errors before and after correction were determined by comparisons with the actual length of $30 \mathrm{~mm}$.

A custom-designed correction model plate shown in Fig. 5-(1) (3) was used to correct the images. Steel balls of a diameter of $2.5 \mathrm{~mm}$ were evenly distributed over the correction plate as feature points (Fig. 5(1)), which was fixed on the end of the image intensifier (Fig. 5-(2)). The steel balls could be clearly imaged on fluoroscopy (Fig. 5-(3)). By measuring the original coordinates of the feature points, a distortion-correction model was built based on the ideal distribution of the feature points, and this model was mapped to the ideal image through geometric transformation (Fig. 6).

All five images were corrected by this method, and the lengths of $A D, D G, B E, E H, C F$, and FI before and after correction were calculated. For the images before and after correction, nine axis pixels of points A-I were selected. Using SolidWorks software(SolidWorks ${ }^{\circledR}$ Premium $2017 \times 64$ version SP2.0, Dassault Systemes), three feature points on each Kirschner wire (ABC, DEF, GHI) were fitted to a circle, and the radius of each circle was calculated, with these radii being used to evaluate the correction. The larger the radius is, the smaller the curvature is, and the closer the points were to being on a straight line.

\section{Results}

\subsection{Data analysis of the sacroiliac fixation procedures}

The biplanar robot was used to locate the sacroiliac nailing channel for the four patients, and the individual results are described below.

\section{Case 1}

The guide pin location shown on the pelvic outlet film demonstrated little variation, which was acceptable (Fig. 1.1, 1.2). However, the anterior edge of the sacrum was located in the image center on the pelvic positioning inlet film, as shown in Fig. 1.3, while the center of most images were inclined to the pelvic center, and the anterior edge of the sacrum was inclined to the top of the image (Fig. 1.4). Moreover, after the guide pin was placed following the nailing positioning channel (Fig. 1.3), the actual guide pin position (Fig. 1.5) showed a dorsal displacement. Compared with the 2.8-mm guide pin, the actual guide pin position should be about $4 \mathrm{~mm}$ further back.

Although there was some deviation from the preoperative planning, the actual nailing channel was acceptable for the clinical work, and thus it was not adjusted, and the operation was continued.

\section{Case 2}


In this case, the pelvic projection angle had a little rotation, such as on the outlet positioning film (Fig. 2.1), which had a certain clockwise rotation, while some had an anti-clockwise rotation (Fig. 2.2). In the whole process, the position of the projection center was relatively fixed (Fig. 2), and the final guide pin position was basically the same as that of the preoperative positioning of the nailing channel (Fig. 2.3 and 2.6).

\section{Case 3}

After locating the nailing channel with the positioning films (Figs. 3.1 and 3.4), the operator used just the inlet image (Fig. 3.2) to verify the guide pin placement. As the second sacral guide pin was penetrated, its position was about $3 \mathrm{~mm}$ upward and close to the first sacral hole (shown in Fig. 3.5). If the second sacroiliac screw was inserted following this channel, it could have easily cut out the sacral hole and injured the first sacral nerve. Therefore, the operator discontinued the navigation procedure and manually placed the guide pin (Figs. 3.3 and 3.6). The subsequent position of the second sacral guide pin was acceptable.

\section{Case 4}

In this case, the nailing channel on the inlet and outlet positioning films was relatively central (Fig. 4.1 and 4.3). During the navigation procedure, the entrance of the guide pin was right on the point, but the penetration direction suffered an external rotation of about $5^{\circ}$. When the tip of the guide pin reached the first sacral vertebra, it was very close to the front edge of the vertebra (Fig. 4.4), and the tip of the guide pin was about 2.5-mm forward. The position was acceptable after manual implantation (Figs. 4.5 and $4.2)$.

\subsection{The observation of respiratory motion in the biplanar navigation system}

Under general anesthesia and tracheal intubation, five consecutive fluoroscopic films at the pelvic inlet and outlet positions were acquired at intervals of 2-3 s. The two films with the sharpest contrast were selected and merged for comparison, and are shown in Fig. 7, with the green area being near the exhalation end and the pink area near the inhalation end. As shown in Fig. 7, several important anatomical structures of the pelvis had a slight caudal displacement. The large ischial notch marked by the red line had a caudal displacement of about $3 \mathrm{~mm}$, and the sacrum promontory marked by the yellow line had a caudal displacement of about $5 \mathrm{~mm}$, which shows a sharp contrast.

\section{The Error Model Verification Experiment}

Using the biplanar method, the spatial positioning error results for the K-wire tip are shown in Table 1. The positioning accuracy of the system was within $2 \mathrm{~mm}(1.60 \pm 0.28 \mathrm{~mm}$ for the entrance point; $1.28 \pm$ $0.43 \mathrm{~mm}$ for the exit point). 
Table 1

Spatial positioning error results of the error model verification experiment

\begin{tabular}{|c|c|c|c|c|c|c|c|c|c|}
\hline \multirow[t]{2}{*}{ No. } & \multirow[t]{2}{*}{ value } & \multicolumn{3}{|c|}{$\begin{array}{l}\text { Coordinate (entrance } \\
\text { point) }\end{array}$} & \multicolumn{3}{|c|}{ Coordinate (exit point) } & \multirow{2}{*}{$\begin{array}{l}\text { Error } \\
\text { (entrance } \\
\text { point) }\end{array}$} & \multirow{2}{*}{$\begin{array}{l}\text { Error } \\
\text { (exit } \\
\text { point) }\end{array}$} \\
\hline & & $x$ & Y & Z & $x$ & $\mathrm{Y}$ & Z & & \\
\hline \multirow[t]{2}{*}{1} & True value & -20 & 150 & 116.5 & 50 & 147 & 106 & \multirow[t]{2}{*}{1.4872} & \multirow[t]{2}{*}{1.7508} \\
\hline & $\begin{array}{l}\text { Calculated } \\
\text { value }\end{array}$ & -18.58 & 150.27 & 116.15 & 51.25 & 145.98 & 106.68 & & \\
\hline \multirow[t]{2}{*}{2} & True value & -20 & 150 & 116.5 & 50 & 147 & 106 & \multirow[t]{2}{*}{1.3678} & \multirow[t]{2}{*}{0.5270} \\
\hline & $\begin{array}{l}\text { Calculated } \\
\text { value }\end{array}$ & -18.79 & 149.37 & 116.60 & 50.32 & 146.68 & 106.27 & & \\
\hline \multirow[t]{2}{*}{3} & True value & -20 & 150 & 116.5 & 70 & 147 & 106 & \multirow[t]{2}{*}{1.7658} & \multirow[t]{2}{*}{1.6662} \\
\hline & $\begin{array}{l}\text { Calculated } \\
\text { value }\end{array}$ & -18.91 & 148.62 & 116.66 & 69.23 & 146.06 & 107.14 & & \\
\hline \multirow[t]{2}{*}{4} & True value & -20 & 150 & 116.5 & 70 & 147 & 106 & \multirow[t]{2}{*}{1.8795} & \multirow[t]{2}{*}{0.8270} \\
\hline & $\begin{array}{l}\text { Calculated } \\
\text { value }\end{array}$ & -18.60 & 148.94 & 117.17 & 70.60 & 146.82 & 106.54 & & \\
\hline \multirow[t]{2}{*}{5} & True value & -20 & 150 & 121 & 50 & 150 & 111 & \multirow[t]{2}{*}{1.6473} & \multirow[t]{2}{*}{1.4956} \\
\hline & $\begin{array}{l}\text { Calculated } \\
\text { value }\end{array}$ & -20.16 & 149.60 & 119.41 & 49.56 & 149.56 & 109.64 & & \\
\hline \multirow[t]{2}{*}{6} & True value & -20 & 150 & 121 & 50 & 150 & 111 & \multirow[t]{2}{*}{0.9402} & \multirow[t]{2}{*}{1.5786} \\
\hline & $\begin{array}{l}\text { Calculated } \\
\text { value }\end{array}$ & -20.50 & 150.79 & 120.90 & 49.40 & 149.14 & 109.82 & & \\
\hline \multirow[t]{2}{*}{7} & True value & -20 & 150 & 116.5 & 50 & 147 & 106 & \multirow[t]{2}{*}{1.8554} & \multirow[t]{2}{*}{1.2675} \\
\hline & $\begin{array}{l}\text { Calculated } \\
\text { value }\end{array}$ & -19.75 & 149.06 & 118.08 & 50.87 & 146.76 & 106.89 & & \\
\hline \multirow[t]{2}{*}{8} & True value & -20 & 150 & 116.5 & 50 & 147 & 106 & \multirow[t]{2}{*}{1.6599} & \multirow[t]{2}{*}{1.5212} \\
\hline & $\begin{array}{l}\text { Calculated } \\
\text { value }\end{array}$ & -18.90 & 150.42 & 117.67 & 51.21 & 146.08 & 105.94 & & \\
\hline \multirow[t]{2}{*}{9} & True value & -20 & 150 & 116.5 & 50 & 147 & 106 & \multirow[t]{2}{*}{1.7165} & \multirow[t]{2}{*}{1.4550} \\
\hline & $\begin{array}{l}\text { Calculated } \\
\text { value }\end{array}$ & -18.47 & 149.53 & 117.12 & 50.87 & 146.00 & 106.60 & & \\
\hline 10 & True value & -20 & 150 & 116.5 & 50 & 147 & 106 & 1.6740 & 0.7320 \\
\hline
\end{tabular}




$\begin{array}{lllllll}\text { Calculated } & -19.06 & 149.88 & 117.88 & 50.55 & 146.57 & 106.22\end{array}$

\section{The Error Model Calibration Experiment}

Each image was corrected, and the results are shown in Fig. 8. The actual length between the points AD, $\mathrm{DG}, \mathrm{BE}, \mathrm{EF}, \mathrm{CF}$, and $\mathrm{FI}$ was $30 \mathrm{~mm}$, and their corrected lengths in the figure are shown in Table 2, according to the actual scale calculated from the line EF.

\begin{tabular}{|lllllll|}
\hline & AD & BE & CF & DG & EH & FI \\
\hline Before Correction & 30.31934 & 29.97318 & 31.0754 & 30.48966 & 29.78832 & 29.94804 \\
\cline { 2 - 7 } & 30.29193 & 30.13214 & 31.17631 & 30.7835 & 29.77738 & 29.75867 \\
\cline { 2 - 7 } & 30.42098 & 30.10331 & 31.2658 & 30.72384 & 29.77758 & 29.74996 \\
\cline { 2 - 7 } After Correction & 30.22807 & 29.8129 & 30.98939 & 30.5183 & 29.64763 & 29.64229 \\
& 30.38935 & 29.72951 & 31.12358 & 30.79583 & 29.71189 & 29.83259 \\
& 30.12032 & 30.10541 & 31.11387 & 29.92054 & 29.74747 & 29.56254 \\
& 30.04177 & 30.02222 & 30.72576 & 30.17776 & 29.67161 & 29.84276 \\
& 30.01488 & 30.32128 & 30.67715 & 30.14824 & 29.67733 & 29.67077 \\
\hline 2.93683 & 30.07156 & 30.5282 & 30.21403 & 29.91816 & 29.63413 \\
\hline & 30.14968 & 30.27688 & 30.86446 & 30.13339 & 29.99948 & 29.85175 \\
\hline
\end{tabular}

Table 2

Distance between $A D, D G, B E, E H, C F, F I$ before and after correction $₫ 150 \rrbracket 200 \rrbracket 250 \rrbracket 300 \rrbracket 350 \mathrm{~mm}$ from the receiver respectively $\rrbracket$

For the images before and after correction, three axis pixels were selected on each line (ABC, DEF and $\mathrm{GHI}$ ) and were fitted to a circle with the measuring tools of the SolidWorks software in the five groups of images. The corresponding radius of each circle was then calculated. The results are shown in Fig. 8 and Table 3. 
Table 3

Before and after correction, 3 axial pixels were fitted into a circle with SolidWorks measurement tools and the radii of the circus were used to evaluate the correction

\begin{tabular}{|lllllll|}
\hline \multicolumn{3}{|l}{ Before correction } & \multicolumn{4}{l|}{ After correction } \\
\cline { 2 - 7 } & AC & DF & GI & AC & DF & GI \\
\hline 150 & 14481.39 & 26739.88 & 4254.84 & 34413.10 & 39310.41 & 40407.65 \\
\hline 200 & 17439.19 & 46232.34 & 11027.88 & 20831.31 & 636788.27 & 20860.48 \\
\hline 250 & 13869.60 & 51869.98 & 13133.94 & 79873.08 & 103116.30 & 49029.20 \\
\hline 300 & 14728.76 & 41600.51 & 13503.26 & 51358.42 & 289319.51 & 988794.78 \\
\hline 350 & 111.87 .65 & 48134.78 & 11658.19 & 69833.38 & 118527.53 & 114006.48 \\
\hline
\end{tabular}

\section{Discussion}

With the development of CAS, researchers and surgeons have mainly focused on the advantages brought by this technology, such as high precision, low radiation dose, and a steep learning curve, but little work has focused on the defects of this technology. The image drift error common in navigation technology is very obvious, but a large number of subtle error sources from the CAS technology itself may be ignored by researchers and surgeons, which could accumulate to a large image drift and result in serious consequences. This paper mainly discusses the technical defects of the image intensifier and some small errors caused by respiratory motion during operations using a biplanar navigation system.

\section{Conical distortion drift caused by the technical defects of the image intensifier}

Because of the influence of the image intensifier structure, the imaging principle, imaging environment and the projection can lead to a larger image distortion than that present when an ordinary camera is used. The traditional X-ray fluoroscopy system consists of an X-ray transmitter and a cylindrical receiver (Fig. 12 = 9) that contains the XRII system. In the imaging process, the X-ray beam is emitted from the Xray source and passes through the sample or the human body, and is then converted into visible light through the XRII system, which is projected onto the CCD camera. The image distortions include a pillowshaped distortion caused by the spherical nature of the input screen surface of the X-Ray Image Intensifier (XRII) system, S-shaped distortion caused by the terrain and the environmental magnetic field, and local distortion caused by the installation and manufacturing errors (Fig. 5-a c).

Position and posture variations used to describe the spatial pose of a target are expressed by the translation and rotation on three coordinate axes in the 3D coordinate system (namely, the six degrees of freedom). Different target pose can project different 2D images with the fluoroscopy system. When two 
positioning images are taken from different projection angles with the biplanar navigation system, the errors caused by image distortion can overlap. In addition, the X-rays of the fluoroscopy system follow a conical dispersion pattern, and therefore points from different planes present different dispersion trends from the Z-axis.

\section{Plane dispersion characteristics caused by the conical distortion}

There are two kinds of dispersion characteristics caused by the conical distortion: point distortion dispersion and line distortion dispersion.

Point conical distortion dispersion is shown in Fig. 9. The distance from point $A$ and point $B$ to the Z-axis is equal, and these two points present a line $A^{\prime} B^{\prime}$ on the screen. Point $A^{\prime}$ is a little further from the $Z$-axis than point B'. The point conical distortion has two characteristics. First, the closer the points are to the spherical tube, the more obvious the dispersion trend is and the farther the images are from the Z-axis (A, 'A' point in Fig. 9), and vice versa (B, 'B' point in Fig. 9). When a Kirschner pin is filmed with the fluoroscopy system, the end that is close to the spherical tube will present a wider image than the other one. Second, all the points on the same (perpendicular to the Z-axis) plane would have the same dispersion trend, and the projection image would not present an obvious distortion. This could be called the dispersion feature for the point conical distortion. The point spatial errors shown in the error model verification experiment (Table 1) are in accordance with the dispersion features for the point conical distortion, and there is no obvious spatial distortion error.

There are two types of dispersion features for the line conical distortion. First, when a line AB (Fig. 12) is coplanar with the Z-axis, i.e., they intersect or are parallel, the line can be presented as a straight line on the screen. This situation is shown in Fig. 9. When the longitudinal axis of the Kirschner pin passes through the Z-axis, they are coplanar, and the projection of the Kirschner pin can be presented as a straight line on the screen, which is the first type of dispersion feature for the line conical distortion.

With the second type of dispersion feature, when the line AB and Z-axis are neither parallel nor intersect (that is, they are noncoplanar), an irregular arc would appear on the screen (Fig. 9). There are two causes for this situation. First, the dispersion trend of the points from different planes of the image intensifier is different. Second, because of the influence of the XRII system, the dispersion trend decreases as the distance between the points on the line $A B$ and $Z$-axis decreases. The image formed by the line on the screen would form an arc to the projection center (Z-axis, Fig. 9). This is the second type of dispersion feature for the line conical distortion.

This kind of image distortion is rather small and can be easily ignored. In the biplanar navigation system, the errors of the two positioning films can overlap, which might have a significant effect on the operation results. 


\section{The plane dispersion features of a solid structure caused by conical distortion}

When the biplanar navigation system was used for nailing positioning, the channel structure for the screw placement was always irregular as the nailing channel was positioned for the four patients, but the channel structures could be idealized as cylinders. When the channel structure is horizontally placed on the projection plane, a relatively standard image can be formed, as the dispersion trend of each point is the same, conforming to the dispersion feature for the point conical distortion.

When the cylinder CD is obliquely placed in the projection area of the image intensifier (Fig. 9), the Z-axis passes through its center, which is in accord with the first type of dispersion feature for line conical distortion. Apart from the $C$ end being a little wider than the $D$ end, the overall dispersion trend of the structure shows little change. When the cylinder CD is translated along the $Y$-axis as one the degree of freedom movement, the projection image would change as shown in the difference from Fig. 9-1 to Fig. 93. However, the projection image of the sideline of cylinder CD would change the arc direction during the translation (from Fig. 9-1 to Fig. 9-3), with the arc being to the Z-axis. This change could reflect the second type of dispersion feature for the line conical distortion.

The dispersion features above are also verified through the error model verification and correction experiments. The positioning accuracy can be controlled to within $2 \mathrm{~mm}$, as can the spatial positioning error for the points (entrance point, $1.60 \pm 0.28 \mathrm{~mm}$; exit point, $1.28 \pm 0.43 \mathrm{~mm}$ ). Although these results are clinically acceptable, the effect of the distortion from the image intensifier is mainly reflected in the $2 \mathrm{D}$ image, and the positioning error of a single point has no practical significance.

In the error analysis between the pre- and post-correction images, compared with the distances of AD, DG, $\mathrm{CF}$, and $\mathrm{FI}$ close to the edge of the image, the distances of $\mathrm{BE}$ and $\mathrm{EH}$ close to the Z-axis are generally smaller. This situation presents as pillow-shaped distortion caused by the spherical nature of the input screen surface of the XRII system. The points on the same line may all be distorted, which can affect the curvature of the line, and the distance of the points may not reflect the extent of the line correction; therefore, the concept of curvature (the reciprocal of radius) is introduced to present the correction extent.

First of all, we emphasize that no matter how precise the correction of the image distortion is, the corrected lines AC, DH, and GI can infinitely approach to a straight line, but can never be corrected to a real straight line, and therefore the curvature of the line can well reflect the correction extent of the distortion. That is, the larger the radius is, the smaller the curvature is, and the closer the corrected line is to a real straight line. As shown in Table 3, the radius of the curve representing each line obviously increases after correction, and therefore, as the curvature decreases, the lines become closer to a straight line. The closer to the Z-axis (line DH), the larger the radius of the fitted circle is, the smaller its curvature is, and the higher the extent of the distortion correction. This is the exact presentation of the second type of dispersion feature for the line conical distortion. For example, the curvatures of the line DH before and after correction are both smaller than those of lines AC and GI on both sides. It should be noted that: 1) 
all the images are distorted in the projection process; 2) no matter how precise and rigorous the technology is for the image correction, the distortion cannot be thoroughly corrected to an ideal straight line, and can just be corrected to be infinitely close to one.

\section{The clinical characteristics and the avoidance principle of the conical distortion drift}

During the minimally invasive pelvic fixation procedure, the nailing channel is rarely selected as the projection center. For each projection, the spatial position and posture of the nailing channel is subject to the second type of dispersion features for the line conical distortion. In particular, the safe nailing channel for sacroiliac screws has a special angle.

How can we minimize the error caused by this kind of distortion (Fig 12)?

1. Try to locate the whole projection structure on the same plane perpendicular to the Z-axis, which can be in accordance with the dispersion features for the point conical distortion. The dispersion trend of all parts is basically the same, and there is no obvious image distortion for the projection image.

2. If the position and posture of the projection structure are not in accordance with the dispersion features for the point conical distortion, it is better to locate the longitudinal axis of the projection structure so that it is coplanar with the Z-axis of the image intensifier so that the longitudinal axis direction of the projection structure is consistent with the normal structure. This situation would then be in accord with the first type of dispersion feature for the line conical distortion.

3. If the position and posture of the projection structure are not in accord with the above two features, only the second type of dispersion feature for the line conical distortion is present, and the operators' experience is needed to avoid error.

\section{The effect of the respiratory motion on the navigation precision}

As respiratory oscillation can cause abdominal pressure to fluctuate, the pelvis has a slight pronation and caudal displacement at the end of inhalation. In the example in this study (Fig. 7), it was found that the caudal displacement of the sacral promontory was more than $5 \mathrm{~mm}$, which would not be acceptable in clinical practice, and might cause serious iatrogenic consequences. Therefore, this is a special drift error, and the real-time position and posture of a target such as the pelvis could oscillate at any time. After the marker is registered, the target's spatial position and posture registered in the computer is fixed. The respiratory oscillation would then form a random error, which can be called the random drift. 
If the operator does not pay attention to this problem during the navigation procedure, the biplanar positioning films may be taken as the patient is at the end of inhalation. When the nailing placement is at the end of expiration, the following pelvic oscillation may cause the fixation channel to go through a similar oscillation, and there is the possibility of an unforeseen injury.

However, this problem can easily be solved. During the navigation procedure, the patient is under general anesthesia and endotracheal intubation. Three time phases are chosen for the inlet and outlet positioning films being taken and guide pin penetration. At these three time phases, the ventilator is suspended for a short time under the premise of security at a special respiratory phase (such as the end of the inhalation), then the pelvis is located at the same spatial position and posture and the influences for the respiratory motion can be limited in the special respiratory phase and the operation would result in no serious iatrogenic consequences.

\section{Conclusions}

Although these two kinds of errors can also be seen in the traditional application of fluoroscopy, real-time perspective verification may not be affected by these errors. However, during the biplanar navigation procedure, these errors might affect the surgical results. The errors from both situations are relatively small, and the errors in the navigation operation are the cumulative results of them. Therefore, they may be difficult to separate. Because of the technical limitation of the image intensifier, there is no possibility to completely eliminate these kinds of errors. However, by standardizing the whole operation, the errors can be minimized.

\section{Abbreviations}

CAS: computer-aided surgery; PVC: Polyvinyl chloride; CCD: Charge-coupled Device; 3D: three dimensions; 2D: two dimensions; XRII: X-Ray Image Intensifier

\section{Declarations}

\section{Ethics approval and consent to participate}

All the data and materials involved in the study is available. All procedures performed in this study involving human participants were in accordance with the ethical standards of the committee of the Chinese PLA General Hospital and with the 1964 Helsinki declaration and its later amendments or comparable ethical standards. Informed consent was obtained from all individual participants included in the study.

\section{Availability of data and materials}

The datasets used and analyzed during the current study are available from the corresponding author on reasonable request. 


\section{Competing interests}

The authors declare that they have no competing interests.

\section{Funding:}

This study was funded by National Key R\&D Program of China (2017YFB1304201), China Postdoctoral Science Foundation Grant (2019M653941), the National Natural Science Foundation of China (61771489) and Capital Specialized Program (z171100001017198). The authors have full control over all the data. The study will not be published elsewhere in any language without the consent of the copyright owners.

\section{Authors' contributions}

ZLH, TPF and TJD made a contribution in designing the study and providing critical revisions to this article. DHL and HN were responsible in writing the article. YGX and LHS made a contribution in collecting the data. ZGZ and ZSW made a contribution in collecting the data. All authors read and approved the final manuscript.

\section{Acknowledgements}

The authors are grateful to all of the authors of the studies included in the present study and their study participants.

\section{References}

1. Zheng G, Nolte LP. Computer-Assisted Orthopedic Surgery: Current State and Future Perspective. Frontiers in surgery 2015; 2: 66.

2. Giulianotti PC, Coratti A, Angelini M, Sbrana F, Cecconi S, Balestracci T et al. Robotics in general surgery: personal experience in a large community hospital. Archives of surgery 2003; 138: 777-84.

3. Ahlering TE, Skarecky D, Lee D, Clayman RV. Successful transfer of open surgical skills to a laparoscopic environment using a robotic interface: initial experience with laparoscopic radical prostatectomy. The Journal of urology 2003; 170: 1738-41.

4. Menon M, Tewari A, Baize B, Guillonneau B, Vallancien G. Prospective comparison of radical retropubic prostatectomy and robot-assisted anatomic prostatectomy: the Vattikuti Urology Institute experience. Urology 2002; 60: 864-8.

5. Kang SW, Lee SC, Lee SH, Lee KY, Jeong JJ, Lee YS et al. Robotic thyroid surgery using a gasless, transaxillary approach and the da Vinci S system: the operative outcomes of 338 consecutive patients. Surgery 2009; 146: 1048-55.

6. D'Annibale A, Morpurgo E, Fiscon V, Trevisan P, Sovernigo G, Orsini C et al. Robotic and laparoscopic surgery for treatment of colorectal diseases. Diseases of the colon and rectum 2004; 47: 2162-8. 
7. Weinstein GS, O'Malley BW, Jr., Snyder W, Hockstein NG. Transoral robotic surgery: supraglottic partial laryngectomy. The Annals of otology, rhinology, and laryngology 2007; 116: 19-23.

8. Qureshi S, Lu Y, McAnany S, Baird E. Three-dimensional Intraoperative Imaging Modalities in Orthopaedic Surgery: A Narrative Review. The Journal of the American Academy of Orthopaedic Surgeons 2014; 22: 800-9.

9. Jacofsky DJ, Allen M. Robotics in Arthroplasty: A Comprehensive Review. The Journal of arthroplasty 2016; 31: 2353-63.

10. Overley SC, Cho SK, Mehta Al, Arnold PM. Navigation and Robotics in Spinal Surgery: Where Are We Now? Neurosurgery 2017; 80: S86-S99.

11. Bai L, Yang J, Chen X, Sun Y, Li X. Medical Robotics in Bone Fracture Reduction Surgery: A Review. Sensors 2019; 19.

12. Cerveri P, Forlani C, Borghese NA, Ferrigno G. Distortion correction for x-ray image intensifiers: local unwarping polynomials and RBF neural networks. Medical physics 2002; 29: 1759-71.

13. Forlani C FG. Automatic Real-Time XRII Local Distortion Correction Method for Digital Linear Tomography[C]. Computational Science-Iccs 2001; Proceedings. DBLP: 23-6.

14. Gronenschild E. The accuracy and reproducibility of a global method to correct for geometric image distortion in the x-ray imaging chain. Medical physics 1997; 24: 1875-88.

\section{Figures}




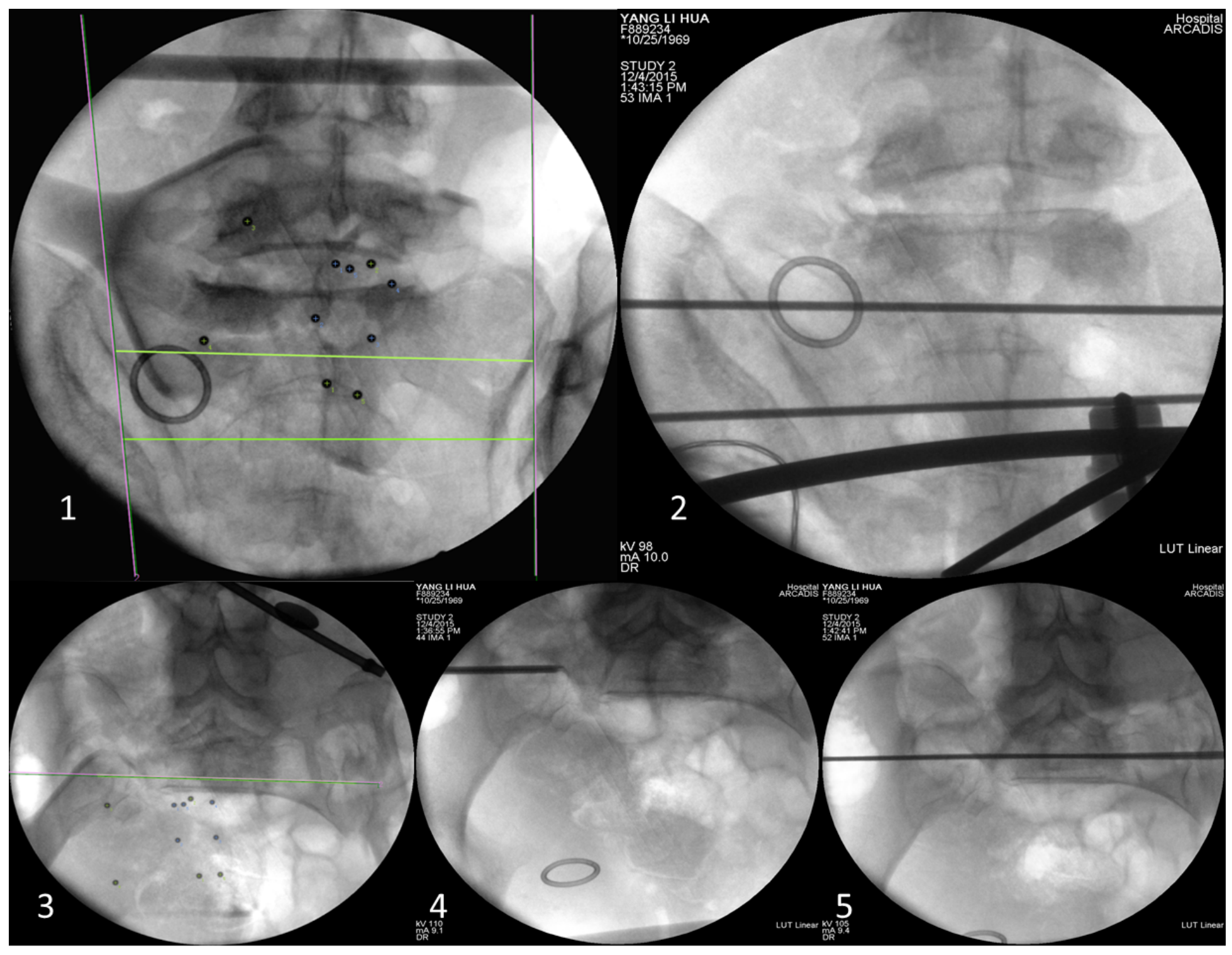

Figure 1

Case 1. The guide pin location demonstrated little variation. After the guide pin placement, its actual position showed a dorsal displacement. The actual nailing channel was acceptable for the clinical work. 


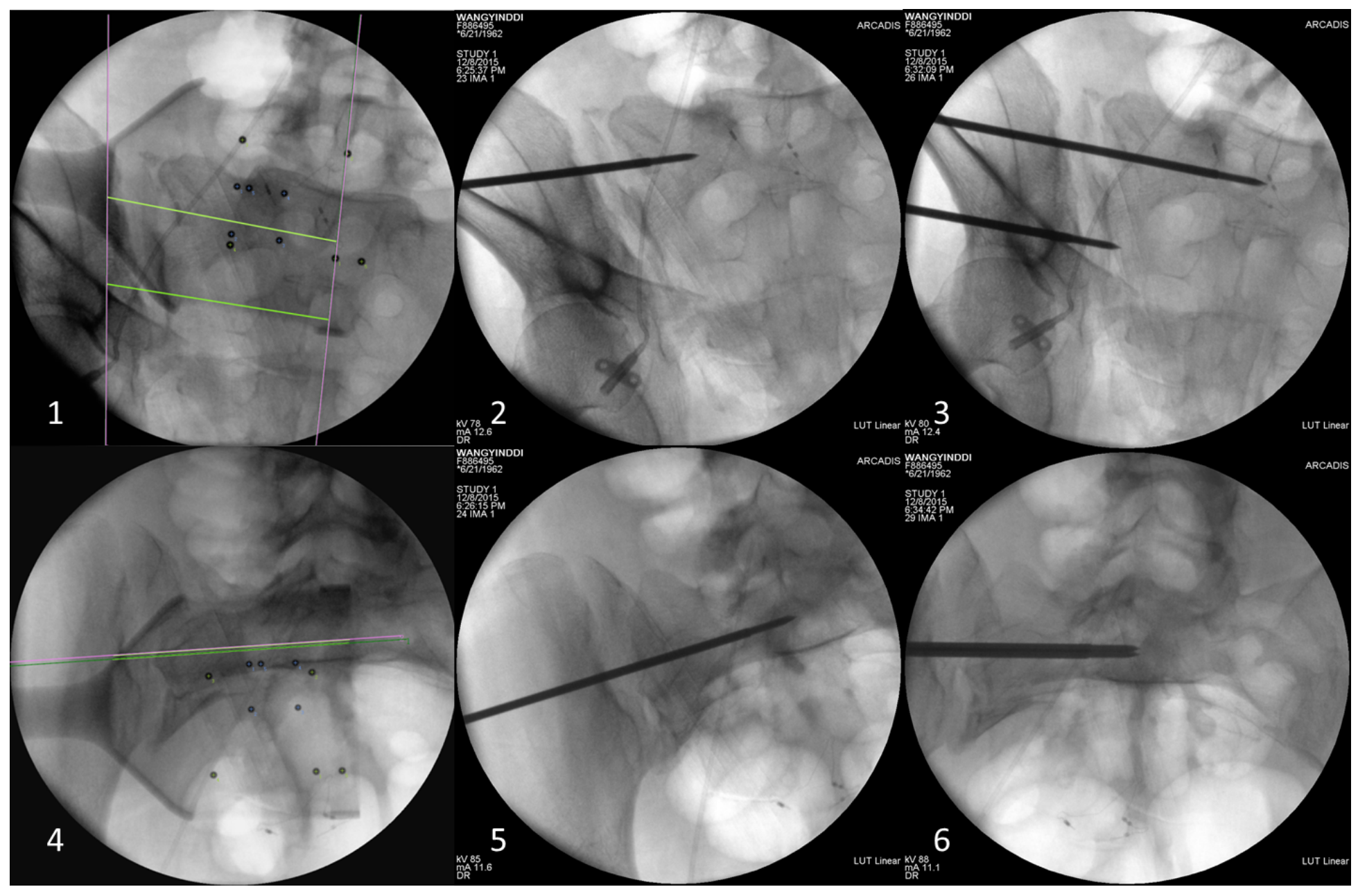

Figure 2

Case 2. The final guide pin position was basically the same as that of the preoperative positioning of the nailing channel. 




Figure 3

Case 3. The position of the second sacral guide pin was about $3 \mathrm{~mm}$ upward and close to the first sacral hole. The operator discontinued the navigation procedure and manually placed the guide pin. The subsequent position was acceptable. 




Figure 4

Case 4. The entrance of the guide pin was right on the point, but the penetration direction suffered an external rotation of about $5^{\circ}$. When penetrating, it was very close to the front edge of the vertebra and was converted to a manual way. 




Figure 5

A C: A rectangular block of $50 \times 80 \mathrm{~mm}$ was manufactured from PVC. (1) (3): A custom-designed correction model plate was used to correct the images. a c: The common image distortions. 


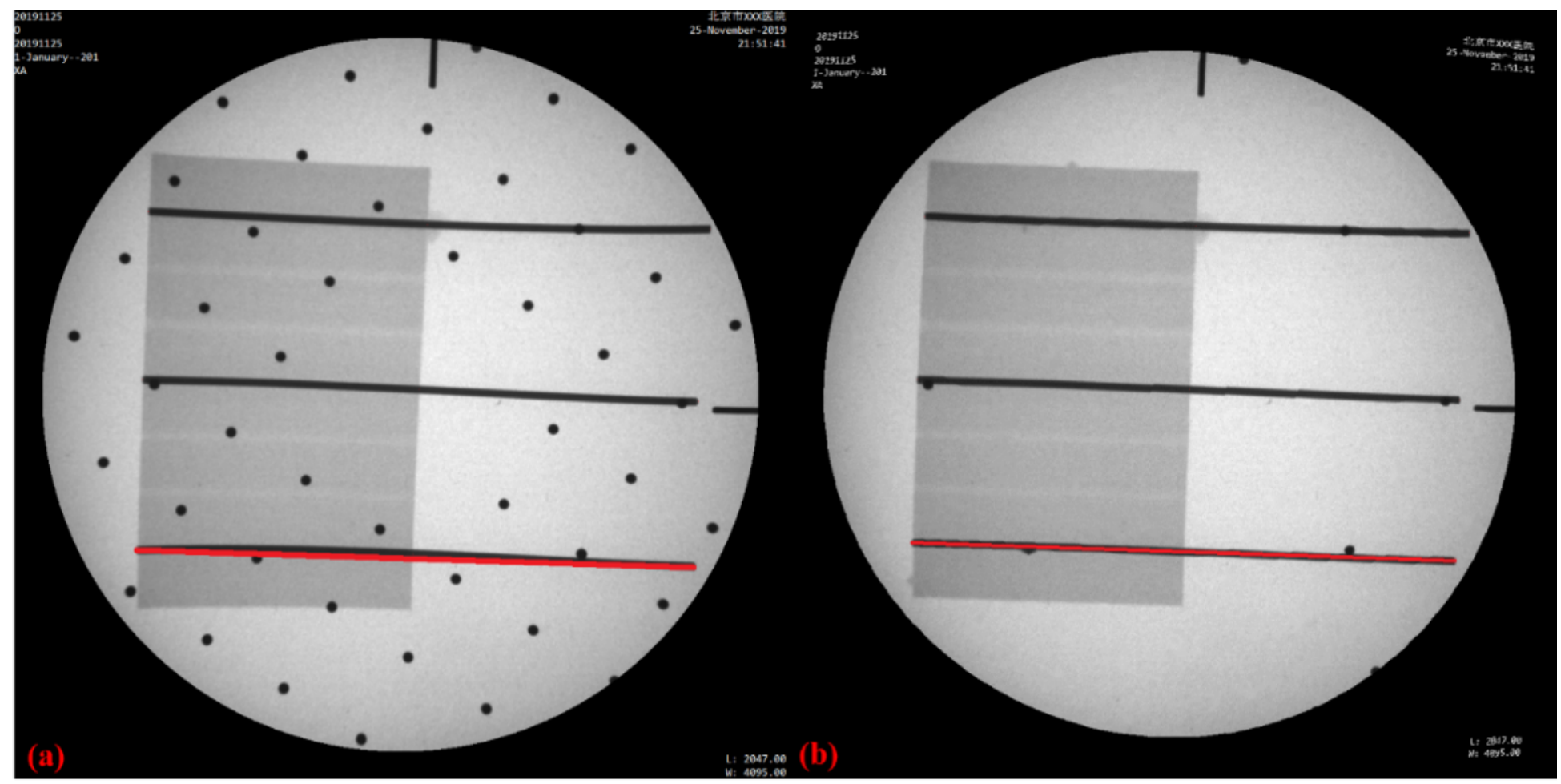

Figure 6

A distortion-correction model was built based on the ideal distribution of the feature points, and this model was mapped to the ideal image through geometric transformation. 
19.09.10-12:53:23-STD

19.09.10-12:53:23-STD-1.3.12.2.1107.5.12.9.152330-September-2019 13:00:20 18-November-1858 0

DR



Figure 7

Two films with the sharpest contrast were selected and merge. The large ischial notch marked by the red line had a caudal displacement of about $3 \mathrm{~mm}$, and the sacrum promontory marked by the yellow line had a caudal displacement of about $5 \mathrm{~mm}$, which shows a sharp contrast. 


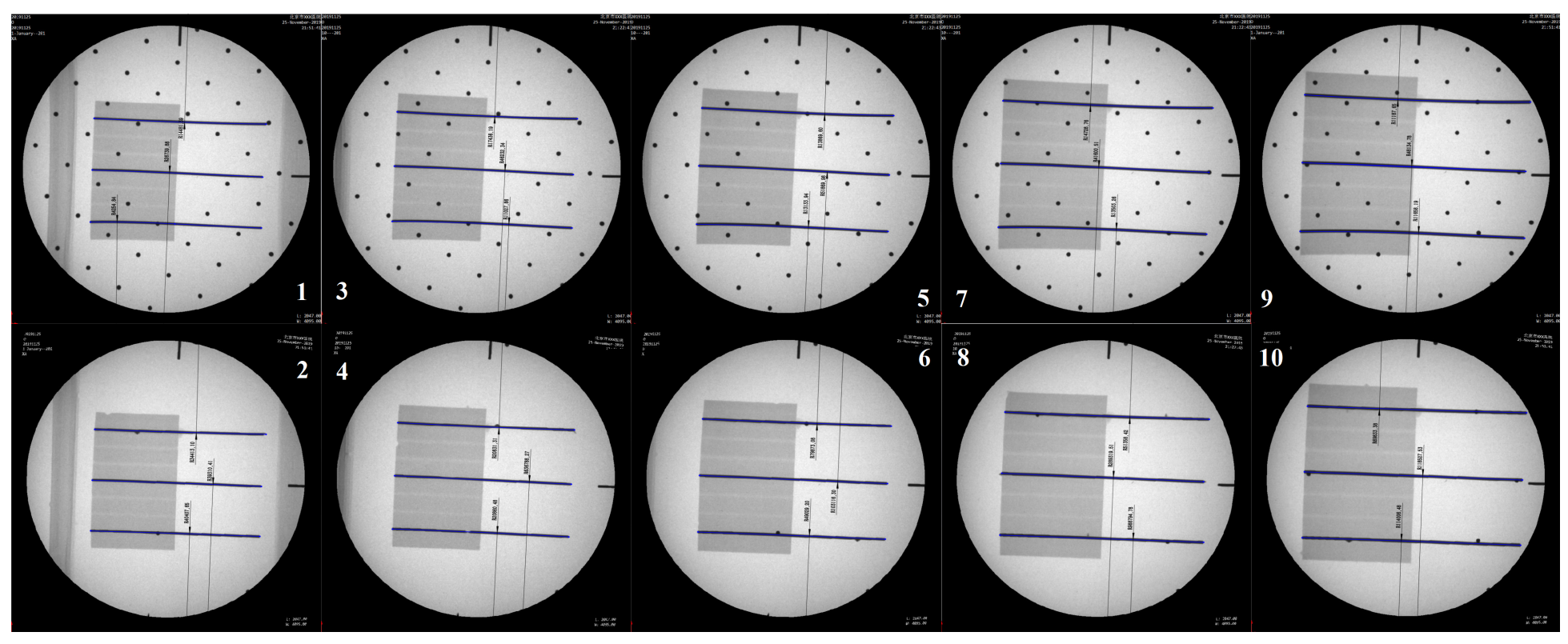

Figure 8

Each image was corrected, and the results are shown. 



Figure 9

The presentations of the dispersion feature for the point conical distortion, two types of the line conical distortion.

\section{Supplementary Files}


This is a list of supplementary files associated with this preprint. Click to download.

- Fig7.mp4 